\title{
Crystal structure of $\mathrm{SiH}_{4}$ at high pressure
}

\author{
Olga Degtyareva, ${ }^{1,2}$ Miguel Martínez Canales, ${ }^{3}$ Aitor Bergara, ${ }^{3,4}$ Xiao-Jia Chen, ${ }^{1}$ Yang Song, ${ }^{1, *}$ Viktor V. Struzhkin, ${ }^{1}$ \\ Ho-kwang Mao, ${ }^{1}$ and Russell J. Hemley ${ }^{1}$ \\ ${ }^{1}$ Geophysical Laboratory, Carnegie Institution of Washington, Washington, DC 20015, USA \\ ${ }^{2}$ Centre for Science at Extreme Conditions, School of Physics, University of Edinburgh, Mayfield Road, Edinburgh EH9 3JZ, United \\ Kingdom \\ ${ }^{3}$ Materia Kondentsatuaren Fisika, Zientzia Fakultatea, Euskal Herriko Unibertsitatea, 644 Posta Kutxatila, 48080 Bilbo, Spain \\ ${ }^{4}$ Donostia International Physics Center (DIPC) and Centro Mixto CSIC-UPV/EHU, 1072 Posta Kutxatila, 20080 Donostia, Spain
}

(Received 12 March 2007; published 31 August 2007)

\begin{abstract}
The crystal structure of a high-pressure phase of silane $\left(\mathrm{SiH}_{4}\right)$, observed between 10 and $25 \mathrm{GPa}$, is solved using powder synchrotron $\mathrm{x}$-ray diffraction and shown to be of the $\mathrm{SnBr}_{4}$ type. The phase is an insulating molecular solid with a monoclinic unit cell containing four tetrahedrally bonded molecules, space group $P 2{ }_{1} / c$. $A b$ initio calculations show the $\mathrm{SnBr}_{4}$-type structure to be favored in this pressure range relative to other recently proposed structures. The fit of the pressure dependence of volume to the Birch-Murnagham equation of state gives the following parameters at ambient pressure if $K_{0}^{\prime}$ is fixed to 4: $V_{0}=250(9) \AA^{3}$ and $K_{0}$ $=7.8(9) \mathrm{GPa}$ for the experimental data, and $V_{0}=255(2) \AA^{3}$ and $K_{0}=6.1(2) \mathrm{GPa}$ for the data obtained from $a b$ initio calculations.
\end{abstract}

DOI: 10.1103/PhysRevB.76.064123

PACS number(s): 61.50.Ks, 62.50.+p, 71.20.-b

\section{INTRODUCTION}

There is a considerable interest in producing metallic, and possibly superconducting, states of solid hydrogen at multimegabar pressures (several hundred gigapascals). ${ }^{1-3} \mathrm{Al}-$ though solid hydrogen becomes opaque in visible light near $300 \mathrm{GPa}$, much higher pressures (e.g., $>400 \mathrm{GPa}$ ) are thought to be needed for metallization, which currently are a great challenge for hydrogen with high-pressure techniques. It has been recently suggested that hydrogen-rich compounds such as $\mathrm{CH}_{4}, \mathrm{SiH}_{4}$, and $\mathrm{GeH}_{4}$ will require pressures far less than expected for pure hydrogen at equivalent densities to enter metallic states. ${ }^{4}$ As is the case for pure hydrogen, these compounds are considered to be good candidates for hightemperature superconductors in their dense metallic forms. ${ }^{4,5}$ Hydrogen can be viewed in these systems as being "chemically precompressed," which lowers the metallization pressure. While the conditions for forming metallic methane have not been reached experimentally, ${ }^{6}$ recent theoretical studies predict that both $\mathrm{SiH}_{4}$ (Refs. 5 and 7) and $\mathrm{GeH}_{4}$ (Ref. 8) transform to a metallic state at much lower and accessible pressures. However, there is only limited high-pressure experimental data available on these compounds, where an evidence for an insulator to semiconductor transition has been reported for solid silane $\left(\mathrm{SiH}_{4}\right)$ at pressures around $100 \mathrm{GPa}$ from optical absorption and reflection measurements. ${ }^{9}$

At ambient pressures, solid $\mathrm{CH}_{4}, \mathrm{SiH}_{4}$, and $\mathrm{GeH}_{4}$ consist of isolated high-symmetry tetrahedrally bonded molecules. For silane, two ambient pressure phases have been reported from the power x-ray diffraction, phase I forming on solidification below $88.5 \mathrm{~K}$ and phase II below $63.5 \mathrm{~K}$. $\mathrm{SiH}_{4}$ molecules form a noncubic crystal structure (probably with a tetragonal symmetry ${ }^{10,11}$ ); however, the exact details could not be determined. Although several competitive crystal structures have been proposed for the high-pressure phases of silane in recent computational studies, $,, 7,12$ there is a complete lack of reliable experimental data for its molecular and crystal structure even at moderate pressures. Such information is important in understanding the changes in the electronic structure leading to metallization. In this paper, we report the crystal and molecular structure of solid silane at high pressures from a combined study using synchrotron $\mathrm{x}$-ray diffraction and first-principles calculations.

\section{EXPERIMENTAL AND COMPUTATIONAL DETAILS}

Electronic grade $(99.998+\%)$ silane $\left(\mathrm{SiH}_{4}\right)$ from Aldrich was used without further purification. Loading was done cryogenically in a dry nitrogen purged glove box by precooling the diamond anvil cell (DAC) in a liquid nitrogen bath. Temperature was measured by a thermocouple attached to the DAC. $\mathrm{SiH}_{4}$ solidified on the cooled rhenium gasket of the cell once the temperature of the cell body was a few degrees lower than the melting point of $\mathrm{SiH}_{4}(88 \mathrm{~K})$. The cell was then sealed and the solid $\mathrm{SiH}_{4}$ was pressurized before warming up to room temperature. The entire loading process was monitored through an optical microscope mounted on the glove box. A ruby chip was inserted into an empty gasket prior to the loading of silane for pressure measurement. The pressure was determined from the shift of the $R_{1}$ ruby fluorescence line with an accuracy of $\pm 0.05 \mathrm{GPa}$ under quasihydrostatic conditions using the ruby scale from Ref. 13. After the loading was complete, the pressure was measured 11.3 GPa. Powder x-ray diffraction data were collected at beam line 16-ID-B (HPCAT) at the Advanced Photon Source. A focused monochromatic beam with wavelength $\lambda=0.3664 \AA$ was used, and the data were recorded on a MAR image plate. The diffraction data were collected at room temperature from 11 to $40 \mathrm{GPa}$ on pressure increase and down to $10 \mathrm{GPa}$ on pressure decrease. Diffraction data were integrated azimuthally using FIT2 $\mathrm{D}^{14}$ and structural information was obtained using JANA2000. ${ }^{15}$

Theoretical calculations were performed within the framework of density functional theory, ${ }^{16,17}$ as implemented by the 


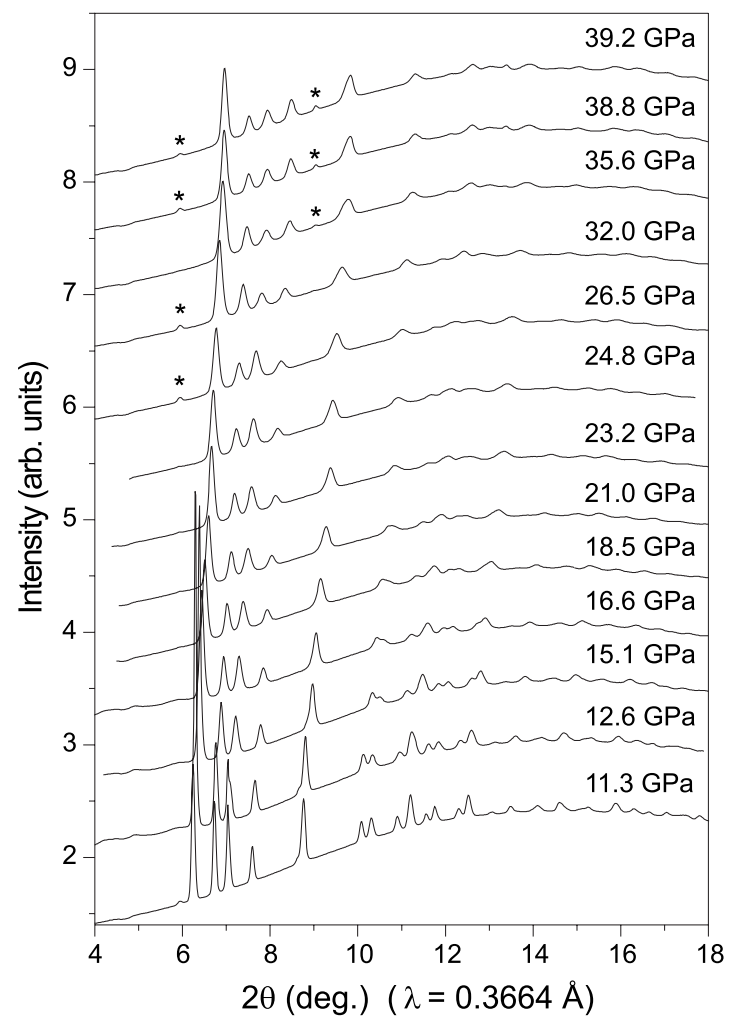

FIG. 1. X-ray diffraction patterns of solid $\mathrm{SiH}_{4}$ at selected pressures. Asterisks denote additional peaks appearing above $25 \mathrm{GPa}$.

VASP package. ${ }^{18,19}$ The Perdew-Burke-Ernzerhof generalized gradient approximation ${ }^{20}$ has been used for the exchangecorrelation functional and, additionally, the all-electron problem has been simplified by means of ultrasoft pseudopotentials. ${ }^{21}$ Plane waves have been chosen as the basis set, and several structures have been studied in the pressure range of interest. For each structure and for every volume, both the lattice parametres and the atomic positions have been optimized via a two step process: Parametres are preoptimized via a low precision relaxation, and this is followed by a high precision relaxation. The final energy has been obtained in a static calculation using a $10 \times 10 \times 10$ Monkhorst-Pack ${ }^{22}$ grid and a $600 \mathrm{eV}$ cutoff. It should be noted that in cases with large length differences between lattice vectors, the grid has been modified accordingly.

\section{RESULTS AND DISCUSSION}

X-ray diffraction of silane at $11.3 \mathrm{GPa}$ revealed a high quality pattern with numerous reflections (Fig. 1). At these conditions, phase V of silane is stable, as found by our Raman and infrared measurements, while silane solidifies at $4 \mathrm{GPa}$, phase III exists in a narrow pressure range from 4 to $6.5 \mathrm{GPa}$, and phase IV is stable from 6.5 to $10 \mathrm{GPa}^{23}$ With pressure increase, the diffraction peaks become significantly broader, but the diffraction pattern does not change significantly. Above $25 \mathrm{GPa}$, additional peaks appear (Fig. $1)$, which signals a phase transformation. The transition pressure of $25 \mathrm{GPa}$ is in agreement with that obtained in our optical measurements. ${ }^{23}$

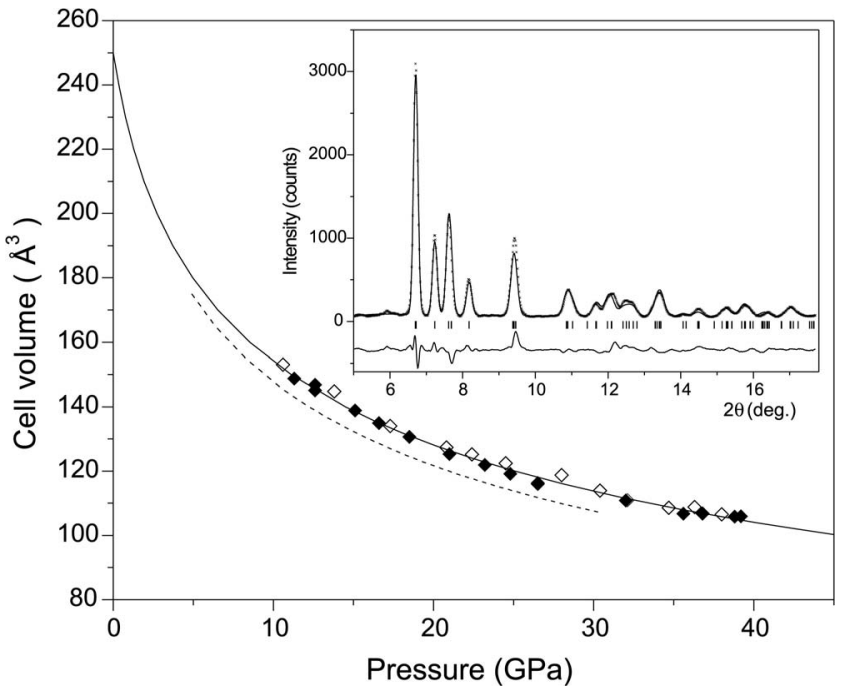

FIG. 2. Pressure dependence of the unit cell volume of the $\mathrm{SiH}_{4}$ phase V. Solid and open symbols show data collected on pressure increase and pressure decrease, respectively. Solid and dashed lines represent the experimental and theoretical equations of state. Inset: integrated profile of the $\mathrm{SiH}_{4}$ phase $\mathrm{V}$ at $24.8 \mathrm{GPa}$ collected on pressure increase (crosses) and the Rietveld refinement fit for the $P 2_{1} / c$ structure (line). The tick marks below the profile show the calculated peak positions. The lower curve is the difference between the observed and calculated profiles.

Phase $\mathrm{V}$ of silane is indexed with a monoclinic cell with lattice parameters $a=6.161(1) \AA, \quad b=4.0722(2) \AA, \quad c$ $=6.122(1) \AA$, and $\beta=104.36(1)^{\circ}$ at $11.3 \mathrm{GPa}$, giving a unit cell volume of $148.78(1) \AA^{3}$ at this pressure. All diffraction patterns up to $25 \mathrm{GPa}$ could be indexed with this unit cell. Above this pressure, when the additional peaks appear, all the diffraction peaks, except the additional peaks, could still be fitted with the monoclinic cell. This indicates that above $25 \mathrm{GPa}$, the structure of phase $\mathrm{V}$ either becomes more complex or coexists with an appearing new high-pressure phase. The fit of the pressure dependence of the unit cell volume for phase V (Fig. 2) with the Birch-Murnagham equation of state gives the following parameters at ambient pressure: $V_{0}$ $=250(9) \AA^{3}$ and $K_{0}=7.8(9) \mathrm{GPa}$ with $K_{0}^{\prime}=4$ assumed. The estimation using the ambient volume of the $\mathrm{SiH}_{4}$ molecule of $73 \AA^{3}$ gives four molecules in the unit cell. The space group is determined as $P 2_{1} / c$ (No. 14). Phase $\mathrm{V}$ of $\mathrm{SiH}_{4}$ appears to be isostructural with $\mathrm{SnBr}_{4}$ and shares this structure type with several tetrahalides, where the $\mathrm{Si}$ atom is tetrahedrally coordinated by four hydrogen atoms (Fig. 3). Table I summarizes structural characteristics of known $\mathrm{SnBr}_{4}$-type phases in comparison to $\mathrm{SiH}_{4} \mathrm{~V} \cdot{ }^{24-30}$ The $b / a$ and $c / a$ ratios of the $\mathrm{SiH}_{4} \mathrm{~V}$ structure fall within the margins of the axial ratios of the other $\mathrm{SnBr}_{4}$-type structures. The $\beta$ angle of the monoclinic unit cell takes a value of $102.2^{\circ}-104.4^{\circ}$ for all the observed structures and is virtually pressure independent for the $\mathrm{SiH}_{4} \mathrm{~V}$ structure.

The experimental diffraction pattern at $24.8 \mathrm{GPa}$ was fitted on the basis of the $P 2_{1} / c$ structure with $\mathrm{Si}$ atoms placed in the $4 e$ position $(0.249,0.581,0.371)$ and hydrogen atoms at $4 e \quad(0.036,0.571,0.158), \quad 4 e \quad(0.325,0.946,0.434), \quad 4 e$ $(0.453,0.396,0.300)$, and $4 e(0.186,0.395,0.574)$ positions, as 


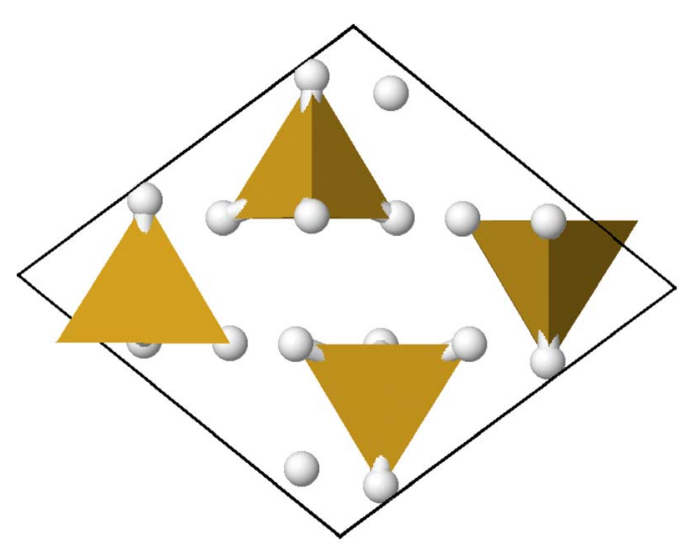

FIG. 3. (Color online) Calculated molecular and crystal structures for the $\mathrm{SiH}_{4}$ phase $\mathrm{V}$ at $24 \mathrm{GPa}$, shown along the $b$ axis.

obtained from our $a b$ initio calculations. As can be seen in Fig. 2 (inset), the positions and intensities of the diffraction peaks of the $\mathrm{SiH}_{4} \mathrm{~V}$ are fitted well within the proposed structures. The resulting $R$ factors from the Rietveld refinement are $R_{p}=12.7 \%$ and $R_{w p}=9.9 \%$. Although the diffraction rings appeared to be relatively smooth, a strong preferred orientation was present. The March-Dollase preferred orientation model has been used to fit the diffraction pattern, which gave the best fit in the $[0,1,-1]$ direction. The refined lattice parameters at this pressure are $a=5.699(1) \AA, b=3.814(1) \AA$, $c=5.643(1) \AA$, and $\beta=104.38(1)^{\circ}$.

The stability of the $\mathrm{SiH}_{4} \mathrm{~V}$ structure is confirmed by our ab initio calculations. According to these (Fig. 4), in the pressure range from 2 to $27 \mathrm{GPa}$ the monoclinic $P 2_{1} / c$ structure is favored over other molecular solids [with $\mathrm{SiH}_{4}$ molecules packed in bcc $\left(\mathrm{GeF}_{4}\right.$-type), simple cubic, and fcc $\left(\mathrm{CH}_{4}\right.$-type $)$ arrangements considered in a recent theoretical work ${ }^{5}$. It is also more stable than the Pmna structure suggested by Feng et $a l .{ }^{5}$ and the $P 2 / c$ structure proposed by Pickard and Needs. ${ }^{7}$ As shown in Table I, a very good

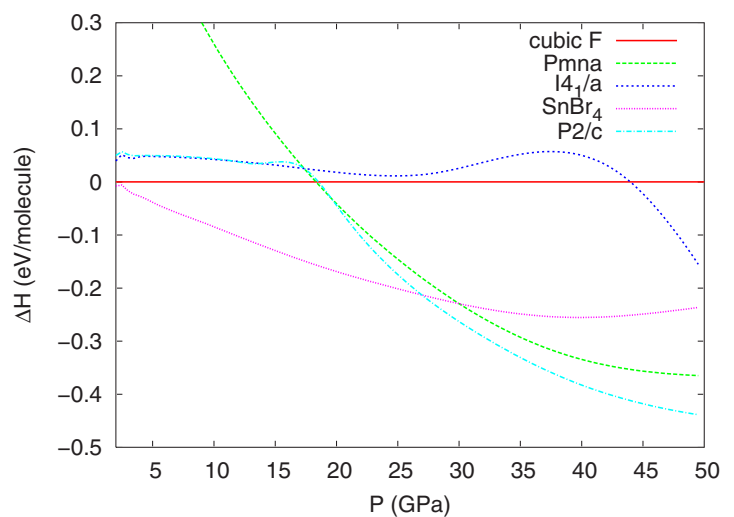

FIG. 4. (Color online) Enthalpy evolution with pressure of the relevant structures of $\mathrm{SiH}_{4}$ considered here. The enthalpy of the face-centered-cubic structure is used as a reference.

agreement between calculated and measured structural parameters defining the $\mathrm{SiH}_{4} \mathrm{~V}$ structure is also obtained. Additionally, the calculated compressibility of the $P 2_{1} / c$ phase agrees well with experiment. Fixing $K_{0}^{\prime}$ to 4 and fitting only calculated data of $\mathrm{SiH}_{4} \mathrm{~V}$ between 5 and $27 \mathrm{GPa}$ to a BirchMurnagham equation of state gives $V_{0}=255( \pm 2) \AA^{3}$ and $K_{0}$ $=6.1( \pm 2) \mathrm{GPa}$. This result compares well with the experimental equation of state (Fig. 2), considering the approximations made for molecular crystals.

$A b$ initio calculations of the evolution of the Si-H distance with pressure provide additional insight into the modification of its bonding properties. At ambient pressure, the $\mathrm{Si}-\mathrm{H}$ bond length in the $P 2_{1} / c$ structure is $1.488 \AA$, which is identical to that of the free molecule. With increasing pressure, this distance decreases, though perhaps not as much as expected. At $27 \mathrm{GPa}$, although the volume has suffered a 2.4-fold reduction, corresponding to a 1.34 factor in linear dimensions, the $\mathrm{Si}-\mathrm{H}$ distance is still $1.466 \AA$ (a reduction of only $1.5 \%$ ). On the other hand, the six $\mathrm{H}-\mathrm{Si}-\mathrm{H}$ angles in the tetrahedra range between $107.3^{\circ}$ and $111.8^{\circ}$, which is close to the correspond-

TABLE I. Structural characteristics of $\mathrm{SiH}_{4}$ phase V compared with those of tetrahalidies with the $\mathrm{SnBr}_{4}$-type structure.

\begin{tabular}{|c|c|c|c|c|c|c|c|c|}
\hline Phase & $P, T$ & $\begin{array}{c}a \\
(\AA)\end{array}$ & $\begin{array}{c}b \\
(\AA)\end{array}$ & $\begin{array}{c}c \\
(\AA)\end{array}$ & $\begin{array}{c}\beta \\
(\operatorname{deg})\end{array}$ & $b / a$ & $c / a$ & Ref. \\
\hline $\mathrm{SnBr}_{4}$ & $293 \mathrm{~K}$ & $10.59(3)$ & $7.10(2)$ & $10.66(3)$ & $103.6(2)$ & 0.670 & 1.007 & 24 \\
\hline $\mathrm{TiBr}_{4}$ & $293 \mathrm{~K}$ & $10.17(2)$ & $7.09(1)$ & $10.41(1)$ & $102.0(2)$ & 0.697 & 1.024 & 25 \\
\hline $\mathrm{TiCl}_{4}$ & $241 \mathrm{~K}$ & 9.70 & 6.48 & 9.75 & 102.67 & 0.668 & 1.005 & 26 \\
\hline $\mathrm{SnCl}_{4}$ & $234 \mathrm{~K}$ & 9.85 & 6.75 & 9.98 & 102.25 & 0.685 & 1.013 & 26 and 27 \\
\hline $\mathrm{SiCl}_{4}$ & $163 \mathrm{~K}$ & $9.608(4)$ & $6.356(2)$ & $9.672(4)$ & 102.91 & 0.661 & 1.007 & 28 \\
\hline $\mathrm{CCl}_{4} \mathrm{III}$ & $\begin{array}{l}1 \mathrm{GPa}, \\
300 \mathrm{~K}\end{array}$ & $9.08(2)$ & $5.764(3)$ & $9.201(3)$ & $104.29(5)$ & 0.635 & 1.013 & 29 \\
\hline $\mathrm{CF}_{4} \mathrm{III}$ & $\begin{array}{c}6.2 \mathrm{GPa} \\
300 \mathrm{~K}\end{array}$ & 6.776 & 4.423 & 6.818 & 102.97 & 0.653 & 1.006 & 30 \\
\hline $\mathrm{SiH}_{4} \mathrm{~V}$ & $\begin{array}{c}11.3 \mathrm{GPa} \\
300 \mathrm{~K}\end{array}$ & $6.122(1)$ & $4.0722(2)$ & $6.161(1)$ & $104.36(1)$ & 0.661 & 1.006 & Expt. \\
\hline $\mathrm{SiH}_{4} \mathrm{~V}$ & $\begin{array}{c}11.6 \mathrm{GPa} \\
0 \mathrm{~K}\end{array}$ & 6.012 & 4.033 & 6.042 & 104.28 & 0.670 & 1.005 & Theory \\
\hline
\end{tabular}


ing angle in a regular tetrahedron $\left(109.47^{\circ}\right)$. Additionally, the band gap decreases rapidly on compression from $6.36 \mathrm{eV}$ at ambient pressure to $2.86 \mathrm{eV}$ at $27 \mathrm{GPa}$. All these features indicate the prevalence of repulsive intermolecular forces in silane under these conditions and that below $27 \mathrm{GPa}$ silane is an insulating molecular solid.

Figure 4 shows that with increasing pressure, both the $P 2 / c$ structure $^{7}$ and the Pmna structure ${ }^{5}$ are favored over $P 2_{1} / c$. Remarkable differences arise when these structures are compared with $\mathrm{SiH}_{4} \mathrm{~V}\left(P 2_{1} / c\right)$. The $P 2 / c$ structure is characterized by zigzag chains of $\mathrm{H}$-bonded $\mathrm{Si}$ atoms along the $c$ axis, while the Pmna structure can be described as sheets of octahedrally coordinated $\mathrm{Si}$ atoms sandwiched between layers formed by $\mathrm{H}$ double sheets. Therefore, around $27 \mathrm{GPa}$ covalentlike structures with a higher coordination number begin to be preferred over $P 2_{1} / c$. However, we remark that the observed x-ray diffraction pattern above $27 \mathrm{GPa}$ (Fig. 1) cannot be reproduced considering either $P 2 / c$ nor Pmna structures with the atomic positions obtained from $a b$ initio calculations. The observed diffraction patterns indicate that $\mathrm{SiH}_{4}$ transforms to a new structure in this pressure range. In this regime, major changes in electronic properties are predicted. ${ }^{5}$

In conclusion, the crystal structure of $\mathrm{SiH}_{4}$ between 10 and $25 \mathrm{GPa}$ has been solved from powder diffraction data and confirmed by $a b$ initio calculations. In this pressure range, $\mathrm{SiH}_{4}$ is found to be a molecular insulator with a monoclinic unit cell containing four tetrahedrally bonded molecules (space group $P 2_{1} / c$ ). The x-ray diffraction patterns observed above $27 \mathrm{GPa}$ are more complex than predicted by the $P 2_{1} / c$ structure and are not compatible with either $P 2 / c$ or Pmna structures. Further experimental and theoretical work is underway to investigate the higherpressure phases.

\section{ACKNOWLEDGMENTS}

The authors thank H. P. Liermann for his help with experiments. This work was supported by NSF (DMR), DOE Grant No. DEFG02-02ER4595, and DOE (CDAC). The x-ray measurements were performed at HPCAT (Sector 16), Advanced Photon Source (APS), Argonne National Laboratory. The HPCAT facility is supported by DOE-BES, DOENNSA (CDAC), NSF, DOD-TACOM, and the W.M. Keck Foundation. The use of the APS was supported by DOE-BES under Contract No. W-31-109-ENG-38. M.M.C. would like to thank the Spanish Ministry of Education and Science for providing a grant. M.M.C. and A.B. acknowledge partial support from the EC sixth Framework Network of Excellence NANOQUANTA (NMP4-CT-2004-500198). Computational resources for this work were provided by the SGI/IZOSGIker at the UPV/EHU (supported by the Spanish Ministry of Education and Science and the European Social Fund).
* Present address: Department of Chemistry, The University of Western Ontario, London, Ontario Canada N6A 5B7.

${ }^{1}$ A. F. Goncharov, E. Gregoryanz, R. J. Hemley, and H. K. Mao, Proc. Natl. Acad. Sci. U.S.A. 98, 14234 (2001).

${ }^{2}$ P. Loubeyre, F. Occelli, and R. LeToullec, Nature (London) 416, 613 (2002); see also H. K. Mao and R. J. Hemley, Science 244, 1462 (1989).

${ }^{3}$ C. Narayana, H. Luo, J. Orloff, and A. L. Ruoff, Nature (London) 393, 46 (1998).

${ }^{4}$ N. W. Ashcroft, Phys. Rev. Lett. 92, 187002 (2004).

${ }^{5}$ J. Feng, W. Grochala, T. Jaron, R. Hoffmann, A. Bergara, and N. W. Ashcroft, Phys. Rev. Lett. 96, 017006 (2006).

${ }^{6}$ M. Martinez-Canales and A. Bergara, High Press. Res. 26, 369 (2006); L. Sun, A. L. Ruoff, C.-S. Zha, and G. Stupian, J. Phys. Chem. Solids 67, 2603 (2006).

${ }^{7}$ C. J. Pickard and R. J. Needs, Phys. Rev. Lett. 97, 045504 (2006).

${ }^{8}$ M. Martinez-Canales, A. Bergara, J. Feng, and W. Grochala, J. Phys. Chem. Solids 67, 2095 (2006).

${ }^{9}$ L. Sun, A. L. Ruoff, C.-S. Zha, and G. Stupian, J. Phys.: Condens. Matter 18, 8573 (2006).

${ }^{10}$ W. M. Sears and J. A. Morrison, J. Chem. Phys. 62, 2736 (1975).

${ }^{11}$ E. Legrand and W. Press, Solid State Commun. 18, 1353 (1976).

${ }^{12}$ Y. Yao, J. S. Tse, Y. Ma, and K. Tanaka, Europhys. Lett. 78, 37003 (2007).

${ }^{13}$ H. K. Mao, J. Xu, and P. M. Bell, J. Geophys. Res. 91, 4673 (1986).
${ }^{14}$ A. P. Hammersley, S. O. Svensson, M. Hanfland, A. N. Fitch, and D. Hausermann, High Press. Res. 14, 235 (1996).

${ }^{15}$ V. Petricek, M. Dusek, and L. Palatinus, The Crystallographic Computing System JANA2000 (Institute of Physics, Praha, Czech Republic, 2000).

${ }^{16}$ P. Hohenberg and W. Kohn, Phys. Rev. 136, B864 (1964).

${ }^{17}$ W. Kohn and L. J. Sham, Phys. Rev. 140, A1133 (1965).

${ }^{18}$ G. Kresse and J. Hafner, Phys. Rev. B 47, 558 (1993).

${ }^{19}$ G. Kresse and J. Furthmuller, Phys. Rev. B 54, 11169 (1996).

${ }^{20}$ J. P. Perdew, K. Burke, and M. Ernzerhof, Phys. Rev. Lett. 77, 3865 (1996).

${ }^{21}$ D. Vanderbilt, Phys. Rev. B 41, 7892 (1990).

${ }^{22}$ H. J. Monkhorst and J. D. Pack, Phys. Rev. B 13, 5188 (1976).

${ }^{23}$ X. J. Chen, V. V. Struzhkin, Y. Song, A. Goncharov, M. Ahart, Z. X. Liu, H. K. Mao, and R. J. Hemley (unpublished).

${ }^{24}$ P. Brand and H. Sackmann, Acta Crystallogr. 16, 446 (1963).

${ }^{25}$ P. Brand and J. Schmidt, Z. Anorg. Allg. Chem. 348, 257 (1966).

${ }^{26}$ P. Brand and H. Sackmann, Z. Anorg. Allg. Chem. 321, 262 (1963).

${ }^{27}$ H. Reuter and R. Pawlak, Z. Anorg. Allg. Chem. 626, 925 (2000).

${ }^{28}$ L. N. Zakharov, M. Yu. Antipin, Yu. T. Struchkov, A. V. Gusev, A. M. Gibin, and N. V. Zhernenkov, Sov. Phys. Crystallogr. 31, 99 (1986).

${ }^{29}$ G. J. Piermarini and A. B. Braun, J. Chem. Phys. 58, 1974 (1973).

${ }^{30}$ D. Shindo, T. Yoshii, Y. Akahama, and H. Kawamura, J. Phys.: Condens. Matter 14, 10653 (2002). 